\title{
Ala Kusreik Ya- Misak Universidad: Construyendo educación propia
}

\section{Ala Kusreik Ya- Misak Universidad:Building their own Education}

\author{
Samuel Asdrubal Avila ${ }^{1} \&$ Yuly Andrea Ayala ${ }^{2}$
}

\begin{abstract}
Resumen
Con la aparición del Concejo Regional Indígena del Cauca (CRIC) en la década de los 70, iniciado el proceso de recuperación de tierras en el Cauca y apoyados en las leyes colombianas que brindan autonomía a los grupos indígenas en el campo formativo básico medio y superior, la educación propia (que parte del reconocimiento de los saberes ancestrales) se convirtió en un tema de suma importancia para las comunidades indígenas del Cauca, y específicamente en el municipio de Silvia existe una manifestación de ello. Allí, desde 2011, comenzó la construcción de una universidad indígena abierta a todas las personas, más allá de su origen. El siguiente artículo describe el modo en que ALA KUSREIK YA-Misak Universidad busca la construcción de un sistema educativo propio que sirva de plataforma en la recuperación de los saberes, identidad y cultura del pueblo Misak ${ }^{3}$ y cómo, para conseguir este propósito, los Misak han vinculado tanto a la arquitectura de la universidad como a los contenidos de la educación que ofrece, su cosmovisión, identidad y pensamiento sobre el ser Misak, basados en los principios de escuchar, ver, pensar y hacer.
\end{abstract}

Palabras clave: Educación propia; indígenas; Tampal kuari; Nakchak; Misak Universidad

\begin{abstract}
With the emergence of the Cauca Regional Indigenous Council (CRIC) in Colombia, during the 1970, as the land reclamation process in Cauca began, and supported by Colombian laws that grant autonomy for indigenous groups in education (elementary and middle school and higher education), "educación propia" or authoctonous education (that acknowledges ancestral native knowledge) became a fundamental issue for the indigenous communities of Cauca. In the municipality of Silvia-Cauca, there is a clear example of this. Since 2011, the construction of an indigenous university began, which is open to all people who wish to become part of it no matter their origin. The following article describes how ALA KUSREIK YA- Misak University seeks to build its own educational system to
\end{abstract}

Tipología: Artículo de investigación científica y tecnológica

Recibido: $21 / 01 / 2017$

Evaluado: $20 / 02 / 2017$

Aceptado: $24 / 02 / 2017$

Disponible en línea: 01/03/2017

Como citar este artículo: Ávila, S. \& Ayala, Y. (2017). Ala Kusreik Ya- Misak Universidad: construyendo educación propia. Jangwa Pana, 16 (1), 54 - 66. Doi: http://dx.doi.org/10.21676/16574923.1956

1. Dr. en Antropología. Docente de planta de la Universidad del Magdalena, Colombia. Correo electrónico: samuelavila77@hotmail.com. ORCID ID: 0000-0002-9979-022X

2. Tesista. Licenciatura en Educación Básica con Énfasis en Ciencias Sociales. Universidad de Cundinamarca, Colombia. Correo electrónico: ninapacari11@gmail.com. ORCID ID: 0000-0002-1358-8558

3. Existe material referente a su cosmovisión y gestiones de lucha (Aranda, et al., 2015; Bonilla 2012; Tunubalá, Pechené 2010; Muelas 2005) a ellos, al taita Trino Morales Tombé por su valiosa colaboración en la investigación y quienes escapan a este apartado, agradecimientos por la labor y resignificación en el ejercicio de difusión como herramienta de memoria, reconocimiento y pervivencia de los pueblos indígenas 
serve as a platform for recovery of the knowledge, identity and culture of the Misak people, and how, to achieve this purpose, the Misak have been intervened both the architecture of the university, as well as the contents that it offers, their worldview and thoughts about being Misak, based upon the principles of hearing, seeing, thinking, and doing.

Keywords: Authoctonous education; Indigenous people; Tampal kuari; Nakchak; Misak University

\section{Introducción}

Conte on la consolidación delCRIC ${ }^{1}$ y larecuperación, que aún no concluye, del territorio ancestral, la educación propia fue priorizada por las comunidades indígenas por su necesidad y pertinencia en la pervivencia de los pueblos indígenas del Cauca para mantener y reproducir la cultura e identidad propia, la visión de mundo y el sentir indígena. Lo anterior es posible a través de la vinculación de los conocimientos de las comunidades indígenas y no indígenas, rurales y urbanas con quienes los Misak han entrado en contacto con los multiples saberes de los antepasados, abuelos, abuelas, mayores, desarrollados a lo largo de la historia Misak y desde los cuales se pone en entredicho la educación homogeneizante promovida por el Estado colombiano:

Esta pérdida, debilitamiento o subordinación de nuestra educación con respecto al modelo educativo hegemónico, ha ido en detrimento no sólo de nosotros mismos sino de la sociedad en general, en tanto que esta situación pasada y presente, ha limitado sustancialmente la oportunidad de compartir y hacer visible el aporte de nuestros saberes y visiones de mundo; que por qué no, pueden constituir una alternativa de solución a muchas de las problemáticas de convivencia y permanencia de

1. El Consejo Regional Indígena del Cauca-CRIC- se constituye el 24 de febrero de 1971 en una asamblea indígena llevada a cabo en el resguardo de Toribío con asistencia de representantes de los resguardos de Toribío, Tacueyó, San Francisco, Jambaló, Guambía, Totoró y Pitayó, junto con campesinos del Frente Social Agrario- FRESAGRO. Posteriormente fue necesaria otra asamblea el 6 de septiembre del mismo año, en la cual se aprobó la plataforma de lucha. A esta asamblea asistieron, además de los ya mencionados, delegados de Paniquitá, Puracé y Poblazón. La conformación del CRIC debe entenderse en el marco de las transformaciones económicas, políticas y sociales de los años sesenta, resultado de la violencia de la década anterior estrechamente ligada a la posesión de la tierra: desde allí se entiende la necesidad de apropiación de la educación como espacio de lucha de los pueblos indígenas (CRIC 2004). los seres humanos en esta tierra" (CONTCEPI, 2013, p.21).

La educación propia es una educación a favor de las comunidades, un ejercicio autónomo y emancipatorio que conlleva el empoderamiento de las mismas. Es la autonomía de planear, regular y decidir alrededor de los sistemas educativos de acuerdo a los proyectos étnicos, traspasando los parámetros establecidos por el Estado y exigiendo el derecho de intervención en su educación (Baronnet, 2009). Significa:

(...) construir nuestra educación recogiendo desde el sentir de nuestro corazón, el gusto por lo nuestro, la alegría que nos da participar en nuestros rituales pero también aprender en el encuentro con los demás. Es mejorar nuestra vida identificando nuestras necesidades y problemas y construyendo nuevos conocimientos y nuevas luces para pensar (CONTCEPI, 2013, p.19).

Dicha educación se desarrolla a partir de interpretaciones y cosmovisiones o visiónes de mundo particulares de cada comunidad, convirtiéndose en una práctica que se construye colectivamente a partir del reconocimiento de los saberes ancestrales, entendidos estos como "los fundamentos de los conocimientos y pensamientos profundos de los pueblos indígenas en equilibrio y armonía con la madre naturaleza" (CONTCEPI, 2013 p.45).

Una estrategia implementada a lo largo del tiempo para fortalecer y dar continuidad al proceso de educación propia fue la creación de las escuelas del CRIC, orientadas bajo el PEB-Pograma de Educación Bilingüe-, de las cuales algunas se mantienen hasta hoy (Baronnet, 2009). Así mismo, durante los años 80 , se le dio curso a la llamada 
"profesionalización" de los maestros indígenas quienes obtuvieron el titulo de Bachilleres pedagógicos (Castillo y Triviño 2008).

Estos procesos se acompañaron con debates acerca de la conveniencia o no de la formación religiosa, debido a que diversas comunidades religiosas de tendencias católica y protestante fueron las encargadas de la educación en las instituciones educativas indígenas caucanas, y en muchas ocasiones dudaban del valor de los saberes tradicionales Misak (Demera, 2005). Luego se pensó también en la consolidación de universidades indígenas. Producto de ello es la UAIIN Universidad Autónoma Indígena Intercultural- en Popayán, y recientemente la ALA KUSREIK YAMISAK UNIVERSIDAD en la vereda Santiago del municipio de Silvia, departamento del Cauca ${ }^{2}$, en el suroccidente de Colombia, donde se dieron a la tarea de construir, literal y figuradamente, una universidad cimentada desde y en el pensamiento indígena, abierta a todo aquel que desee hacer parte de ella ${ }^{3}$.

Vale la pena resaltar que el tema educativo sin la presencia de los colonos se desarrolló en la cultura Misak en la familia, la comunidad y la naturaleza de una forma integral y propicia para la vida; es en la familia donde inicia la educación recibida por padre, madre, abuelos y tios maternos y paternos, los seres que biológica, cultural y espiritualmente los rodean, y en la que los cerros, las lagunas y otros seres transmisores de conocimiento y energía se suman a su concpeto de familia (CONTCEPI, 2013)_, . El nakchak, el fogón, era y sigue siendo parte fundamental: alrededor suyo se ofrecían los consejos y compartían saberes. Sin embargo en 1915 se abrió la primera escuela formal/ oficial? en territorio guambiano (Misak), con pedagogía religiosa basada en la Biblia. Otras escuelas posteriores, como las de Acción Popular

2. Kauka para el pueblo Misak, que quiere decir madre de los bosques y de los espíritus de las aguas.

3. Silvia se encuentra ubicado en la cordillera central colombiana a 2620 $\mathrm{msnm}$. La conforman los resguardos indígenas de Jambaló, Guambia, Kisgo, Pitayó, Quichaya y Tumburao. Cuenta con una población aproximada de 40283 habitantes, de los cuales aproximadamente el 79,8\% se autorreconoce como indígenas, según datos del Plan de desarrollo, estratégico, participativo e intercultural del municipio de Silvia "Unidos por Silvia con Honestidad y Gestión” 2012-2015.
(1947), fueron impulsadas por Radio Sutatenza. La creación de estas escuelas prohibía el uso de la lengua materna y promovía una cultura ajena a la propia. Años después, hacia 1980, con la premisa de "recuperar la tierra para recuperarlo todo", surgió la propuesta del Proyecto de Educación Propia que se ejecutó alrededor de 1995 y tuvo una duración de doce años, y que luego se retomaría en 2015 con el nombre de Reforma Educativa abierta a la interculturalidad (Aranda, L., Perafan, C.,Mayorga, E., Moreno, S, 2015).

Así, el interés porlapresenteindagaciónsurgecuando en el segundo periodo del año 2012 integrantes de la comunidad Misak se desplazaron del Cauca hacia el Municipio de Fusagasugá-Cundinamarca, para compartir la experiencia gestada en Silvia-Cauca. Pensar, en este artículo, acerca de la universidad Misak, responde al interrogante de cómo ha sido, es, se edifican e implementan los ejercicios de educación propia en esta comunidad, al tiempo que permite cuestionar la forma de traslapar estas prácticas en comunidades no indígenas, realizando así un giro no acostumbrado al permitir que el saber indígena alimente el saber no indígena, en este caso específico el relacionado con la educación.

\section{Materiales y métodos}

La investigación fue desarrollada con un enfoque cualitativo. Se revisaron documentos, material bibliográfico y videos encontrados en la web acerca del pensamiento y origen Misak. También se realizó un trabajo en campo de carácter etnográfico que implicó estar en la región en diferentes periodos entre los años 2014 y 2016. Durante estos periodos de residencia en la comunidad tuvieron lugar recorridos por el resguardo (algunos de ellos registrados en fotografías), y conversaciones informales y entrevistas semiestructuradas (registradas en audio) con abuelos, abuelas, profesores y pobladores (jóvenes y adultos) vinculados directamente con la universidad, así como una revisión bibliográfica de estudios sobre la comunidad Misak (los cuales incluyen documentos generados por la propia comunidad) puesto que 
entender la universidad significa necesariamente saber quiénes son los Misak.

La etnografía se desarrolló de manera que pudiésemos plasmar en un documento una visión de la realidad desde la perspectiva Misak, en este caso la relacionada con la creación y consolidación de la universidad. Para ello los esfuerzos estuvieron centrados en la comprensión del pensamiento Misak involucrado en el diseño de la universidad, tanto en la parte academica como en la parte arquitectónica. Esta comprensión significó la exploración del pensamiento mítico y de la visión de mundo en general, siempre de la mano de personas de la comunidad del resguardo La María y de la vereda Santiago en el municipio de Silvia Cauca.

El análisis de lo aprendido, lo observado, lo registrado de manera escrita (diarios de campo), visual y auditiva, junto con el dialogo continuo con compañeros y compañeras Misak para resolver las dudas relacionadas con esos mismos datos, estuvieron enfocados por en lado en apreciar el desarrollo de esta universidad en el tiempo histórico Misak enmarcado en un proceso de lucha por la identidad, la tierra y el establecimiento de una educación propia, y por el otro su concepción y desarrollo en el plano de la cosmovisión, el plano en que se conectan el tiempo presente con la naturaleza y el territorio y la identidad Misak, y las maneras como ello se traduce en los ejes teóricos que fundamentan Ala Kusreik Ya.

\section{Resultados y Discusión}

\section{De la utopía a la realidad de la educación propia}

Es importante resaltar los espacios y luchas sociales e históricas que diversas comunidades indígenas en Colombia vienen gestando de tiempo atrás por la recuperación de su identidad. En el Cauca el ejercicio arrancó con el restablecimiento del territorio Misak y Páez, no como un simple espacio geográfico sino como un territorio en donde se proyecta la existencia indígena que no puede ser separado de su autonomía, gobierno propio, la vida en comunidad y la educación propia (CONTCEPI, 2013; Autoridad ancestral del pueblo Misak, Grupo GEIM y UNICEF, 2009).

Para algunos pueblos indígenas en Colombia la educación propia ha sido una de las estrategias base en la revitalización de las culturas, en el mantenimiento de la identidad a través de la tradición oral, y en la historizacion y comprensión de las distintas luchas que se han librado hacia la defensa del territorio y permanencia hasta hoy como pueblos (CONTCEPI, 2013). Sin embargo este tipo de educación ha sido criticada y relegada por el Estado y por diversas comunidades religiosas, por trasmitir valores contrarios a los deseados para los ciudadanos colombianos; un fenómeno constante desde el siglo XIX, cuando se planteaba la política de que para conseguir progreso y desarrollo era necesaria la emergencia de un nuevo ciudadano (Pedraza, 1999) y un compromiso de las comunidades, especialmente indígenas y afroamericanas, con los valores cristianos. No ha existido, además, una normatividad equitativa que garantice el derecho a la educación y no excluya propuestas como las de la educación propia, entendiéndose ese derecho como la facultad indígena de realizar un trabajo autónomo y emancipador que permita mitigar la exclusión económica y social (Torres, 1974), y las tensiones y crisis provocadas por las nuevas tecnologías, el conflicto armado, y los debates sobre la identidad acerca de lo que es ser indígena en el siglo XXI (Banco Mundial, 2011; Chavez, 2011; De Sousa, 2010).

La educación propia Misak se concibe y empieza desde la preconcepción, que forma parte de las etapas del ciclo de la vida, la cual se refiere al saber orientar el plan de vida con el pensamiento, sentimiento y actuar en función del territorio, la tierra, territorialidad y lo que de allí dependa para la continuidad de la identidad del pueblo (alimentación, vestuario, medicina, etc.). Esto debe articularse con la conducta individual y colectiva, comprendiendo lo que cada decisión implica (Plan de Salvaguarda Misak, 2014, p.14) anclado siempre 
a la familia, en este caso, Misak. Se extiende luego por todo el ciclo de vida de la persona y continúa incluso después de la muerte, resultado de la labor y el legado que deja gracias a la oralidad, donde emprende el viaje espiritual.

Las consideraciones que se pretenden y procuran en, para, y desde la familia Misak se interpretan como la autonomía de decidir y gestar planes de acción que fortalezcan los procesos de continuidad y pervivencia de la identidad, cultura, tradición, sentir y ser. Lo anterior se construye desde propuestas educativas propias elaboradas a partir de las intervenciones de sus integrantes, considerando diversas posturas en las que todas las voces del entorno recobran igual nivel de importancia debido, una vez más, a la concepción de familia y la comunitariedad que "ha caracterizado al movimiento indígena desde sus inicios, permitiendo que se construya una organización en la cual hay interlocución permanente entre zonas y regiones [...] la comunitariedad posibilita, por lo tanto, una interlocución entre política y gobierno" (CRIC 2004, p.25).

Para algunos pueblos indígenas del Cauca y de Colombia la comunitariedad se fundamenta en la necesidad de buscar la unidad como camino hacia el derecho de diseñar su propio modelo educativo, según los requerimientos de sus integrantes, en un acto de apropiación del ejercicio; todo a partir de la soberanía como comunidades indígenas. Para ello se creó el PEC (Proyecto Educativo Comunitario) como una forma de contrarrestar los efectos poco aportantesdelPEI(ProyectoEducativoInstitucional) propuesto por el Ministerio de Educación. El PEC implicó la elaboración de lineamientos desde la dimensión comunitaria, reorientando la dirección de la educación en función de las perspectivas de las culturas y de las comunidades locales. Es decir que expandió y transformó lo institucional desde lo comunitario, ejerciendo una soberanía desde y para la comunidad (CRIC, 2004).

El espacio recuperado por gran parte de los pueblos indígenas del Cauca, en lo referente a la intervención y participación en las decisiones que influyen directamente en el dia a dia de las comunidades, es de gran relevancia cuando se habla de educación propia debido a su fortalecimiento a partir de elementos políticos, organizativos y académicos necesarios para hacer comunidad y establecer relaciones equitativas con el Estado. Esta educación pensada por y para la comunidad crea, recrea, divulga y reafirma la identidad cultural y garantiza la transmisión de valores y principios de vida, así como las formas propias de organización jurídica y socio-política de cada pueblo al escucharse a sí mismas, ejercer su derecho a la autonomía y llevar cada proceso a diversos espacios llámese escuelas, colegios, pueblos, resguardos, etc. (CONTCEPI, 2013). Todo esto es una muestra de la importancia de la intervención, vinculación y unión que han logrado algunas comunidades en defensa del derecho a la participación como actores políticos capaces de contribuir en los asuntos que les conciernen y competen.

La reconstrucción y fortalecimiento de los procesos educativos se enmarcan en la consolidación de los tejidos o sistemas propios de educación, y es tarea de uno de los estamentos de autoridad indígena como lo es la Autoridad Tradicional (en el caso Misak representada en los cabildos conformados por gobernador, gobernador suplente, alcalde, fiscal, secretario, tesorero y alguacil que deben ser miembros de la comunidad indígena y son reconocidos como una organización espiritual $\mathrm{y}$ sociopolítica encargada de controlar el territorio y la autonomía educativa (CONTCEPI, 2013)). El Sistema Educativo Indígena es una red que articula procesos, interrelaciona dinámicas, espacios, tiempos, actores, experiencias, niveles $\mathrm{y}$ modalidades educativas en función del perfil de sociedad y del proyecto de vida, individual y colectivo que se busca. Supone la construcción colectiva a partir del territorio como fuente de vida espiritual y física en la que todos los aspectos se interrelacionan y funcionan de manera integral.

Por consiguiente los ejercicios de retroalimentación, los procesos y construcciones de cada comunidad y la colectividad, y la colaboración de blancomestizos (término que se utiliza en varias comunidades para referirse a la población no indígena), se hacen necesarios. Este proceso en el departamento del 
Cauca, doloroso también por la pérdida de vidas, ha ido creciendo y fortaleciéndose a lo largo de las últimas décadas con la consolidación de estamentos como el CRIC, y permanece en revisión constante para garantizar la soberanía de las comunidades indígenas en el territorio (Bolaños, 2012; Baronnet, 2009).

Dicho compromiso de idear y ejecutar un Sistema Educativo Propio se apoya en la normativa que permite soportar y contrarrestar legalmente la instauración de una educación lineal, segregadora y excluyente. El Decreto Ley 088 de 1976, que decantaría en el Decreto 1142 de 1978, reconoce uno de los terrenos ganados constitucionalmente en el campo educativo al considerar el derecho de los indígenas a tener una educación de acuerdo con sus particularidades que consulte sus experiencias y procesos, y les permita participar en su diseño, evaluación y escogencia de maestros apropiados haciendo que los pueblos indígenas retornen su poder de decisión sobre aspectos trascendentales como la educación (Rojas 1999). Este trabajo constante e inacabado implica la continua transformación del quehacer de la educación tal y como lo muestra la resolución 011 de julio 02 de 2008, con la que se crea una nuevo sistema educativo en Guambia, actualmente Silvia, para el fortalecimiento en materia de enseñanza a partir de la visión Misak; reestructuración que contempla las experiencias recientes como una estrategia para el mejoramiento de la educación propia.

Todos estos esfuerzos se ven hoy reflejados a través de las escuelas y universidades que continúan defendiendo una educación propia donde los fundamentos del proyecto educativo priorizan las enseñanzas que se dan con el ejemplo. Entiéndase que las escuelas deben ser pilares para sostener las recuperaciones de tierras y demás derechos sociales que se reclaman: hacer la selección de sus maestros, impartir una educación crítica que considere lo propio pero también lo de afuera, motivar la participación de toda la comunidad y considerar la importancia de la lengua propia mediante una educación bilingüe (Tattay, 2013).

\section{Una propuesta llamada Ala Kusreik Ya- Misak Universidad}

El pueblo Misak está conformado por 18 cabildos en el territorio colombiano ubicados en los departamentos del Cauca, Valle del Cauca, Huila, Meta, Caquetá y Cundinamarca (Plan de Salvaguarda del Pueblo Misak, 2014, p. 9).

En el resguardo ancestral de Guambia; en los resguardos de Munchique, municipio del Tambo; en Kurakchak, municipio de Cajibio; Bonanza y San Antonio, municipio de Morales; Piscitao y La María, municipio de Piendamó; Ovejas - Siberia, municipio de Caldono; la Reforma, La Gaitana, Nuevo Amanecer y Nam Misak, en los municipios de La Plata y Argentina en el departamento del Huila; y Ginebra en el departamento del Valle (Dagua, Aranda \& Vasco, 2015, p.36).

Cada uno de los resguardos se mantiene en constante lucha por conservar sus costumbres, contrarrestando las dinámicas externas que invitan a adoptar un estilo de vida alejado de la identidad indígena Misak; un ejercicio de resistencia que ha llevado a construir y replantear alternativas que mitiguen el daño a su cultura. Por ello se han creado emisoras, centros médicos y un sistema educativo propio que incluye a la universidad (Cabildo de Guambía, 2007).

La ALA KUSREIK YA- MISAK UNIVERSIDAD es una iniciativa en respuesta a la preocupación de los mayores. Los mayores son los anteriores, los primeros Misak que partieron al otro mundo y tiempo, que pueden acompañar los trabajos, dar consejos, traer las lluvias, guiar las cosechas gracias al hilo del que todos van unidos (Dagua, et al., 2015, p.56). Actualmente se les dice así a las personas que debido a su experiencia se les reconoce sabiduría en el sentido de su compromiso y conocimiento de la cultura Misak. Ellos son conscientes de que ya no se transita el camino que contiene sus huellas, ocasionando la progresiva pérdida de la cultura, identidad y lengua materna,también conocida 
como Namuy Wam, que quiere decir nuestra lengua o Namtrik y refiere a la lengua hablada. Con la implementación del idioma castellano en territorios guambianos, y la necesidad de escribirlo con el fin de participar en las relaciones institucionales con el estado colombiano, se contribuyó al detrimento de la lengua (Aranda, 2001).

Por tal motivo, tanto en el diseño arquitectónico de la universidad como en el contenido curricular, cada detalle vinculado con el fortalecimiento de la cultura propia ha sido tenido en cuenta. Veamos de qué maneras el pensamiento ancestral Misak, un pensamiento ligado al agua, al aroiris y a las montañas, se ve reflejado en ellos:

En cuanto a la estructura el edificio es circular, de una sola planta o piso, coronada por un techo en forma de cúpulas (ver figura 1). El diseño circular reproduce la forma y el contenido simbólico del sombrero Pandereta, Kuarimpoto o Tampal Kuari, usado por los Misak. (El vestuario guambiano es en un elemento muy importante en la identidad de este pueblo (Camelo, 1993).

\begin{abstract}
El anaco o falda representa el espacio, territorio, los límites, las familias entre otras cosas; el sombrero propio que con su forma y utilización de los colores también habla del tiempo que está en un devenir: la dinámica de ir volver, cuando nace un niño su vida se desenrolla, luego llega a la vida mayor y posteriormente a la muerte para volver a enrollarse, proceso que se da tanto en la vida como espiritualmente" (Aranda, et al., 2015, p.49).
\end{abstract}

Sin embargo, no necesariamente toda la comunidad los usa. Hoy algunos adolescentes, hombres y mujeres, se resisten a hacerlo especialmente cuando van a las ciudades. Otros, por el contrario, lo utilizan combinándolo con prendas "de ciudad" u optan por no llevar el sombrero para poder lucir cortes de pelo que se estén usando en el momento).

El sombrero Tampal Kuari es entonces un elemento que trasciende su uso corporal porque es parte integral de la identidad del ser Misak, teniendo en cuenta que le fue dado a la comunidad en tiempos míticos.

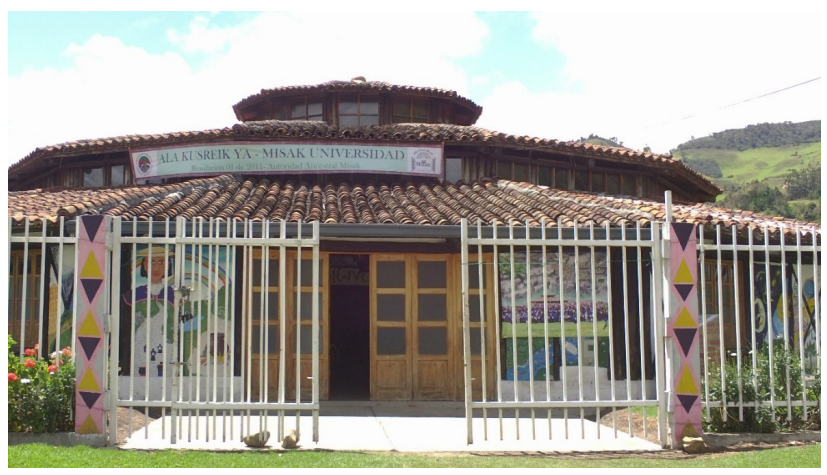

Figura 1. Entrada principal de la ALA KUSREIK YA- MISAK UNIVERSIDAD, que permite observar su diseño en forma de sombrero Tampal Kuari

El mito de origen cuenta que en el principio, junto con la tierra y el agua, se hallaban las lagunas en lo alto de las montañas. La más grande de ellas era Nupisu o Piendamú. De las lagunas descendía el agua hasta encontrarse con el mar y nuevamente las nubes la regresaban en forma de lluvia, para continuar el ciclo. En ese mismo lugar en el que se localizaban las lagunas estaba Pishimisak, que era él y ella a la vez, dueño y cuidador de todo.

Un día, por el agua que se filtraba y removía la tierra ocasionando derrumbes, la tierra se aflojó y se formaron grietas de las que tiempo después nacieron los Pishau, los primeros humanos. Por esto, cada vez que hay un derrumbe, los Misak dicen que el agua huele a sangre porque, como mujer, la tierra está pariendo. Los Pishau son los mismos Misak, criados y alimentados por el Pishimisak, quienes poblaron el territorio incluso llegando a Pupayán antes de la llegada de los españoles. De ahí que los Misak se consideran hijos del agua, del Pishimisak. Hubo otros derrumbes y cada derrumbe trajo consigo un nuevo humano, una nueva autoridad que les enseñaba a trabajar el oro, el tejido, construir herramientas, sembrar. Y del aroiris (los mayores y mayoras del pueblo Misak hablan de aroíris: no es un arco sino un círculo que encuentra su otra mitad bajo la tierra), que nació también del agua y bajó con sus colores por debajo de la tierra, obtuvieron el sombrero, el tampal kuari o kuarimpoto, que teje su historia, su vida y memoria en espiral; un caracol que se enrolla y desenrolla, que vuelve al centro y sigue recorriendo su camino, un eterno retorno, 
aspecto que se puede apreciar en la técnica que se usa para tejer el sombrero (Dagua, et al., 2015).

El sombrero de hojas de pindo o caña brava lo tejen los hombres en forma de caracol con una cinta larga conformada por varias hebras de color rosado, azul claro, azul oscuro y morado. Es significativo para el pueblo Misak porque en él se representa una etapa de la vida de quien lo usa: su niñez, juventud, adultez, si es casado, soltero, viudo, con o sin hijos. Cada sombrero encierra una historia y, de la misma forma que el conocimiento, está en continuo avance, construcción, expansión, transformación y perduración (conversación con el taita Trino Morales Tombé, coordinador académico de la Misak Universidad, septiembre de 2016).

El sombrero también resalta el sentido y relevancia de la comunidad, del pensar en todos, el nosotros, la equidad e igualdad:

Maya es el centro del sombrero propio; en su centro hay uno, un cacique. Ele, va dando todos los colores que rodean el centro, los colores del aroíris. Allí está uno que dirige, que ve todas las cosas. Sale del centro y va girando alrededor, encontrando muchos caminos y organizaciones. Va saliendo pero no sale del todo. Cuando llega al extremo, el cacique Payán va volteando para encontrar otra vez el centro. Y se devuelve por el mismo hilo, el tom, que lo devuelve hasta llegar al mismo centro (Dagua, et al., 2015, p.174).

Maya se interpreta como todos juntos. Ele es el derecho de todos de comer, y tem es el que une. Por ello se dice que el aroíris tiene tem mera: donde se empatan los colores.

El sombrero se convierte en elemento relevante y significativo en la existencia Misak al recordarles el sentido de colectividad, colaboración, unión, el pensar en todos. En el centro del primer círculo del sombrero está un trébol que simboliza el fogón o Nakchak que se encuentra en la mitad de todas las cocinas de las viviendas Misak, y es punto de reunión, de encuentro. Allí se reciben y se renuevan día a día las enseñanzas de abuelos a padres, de abuelos a nietos, de padres a hijos; pasan de generación en generación los legados, principios, derechos y deberes Misak fundados en la autoridad, autonomía, identidad y territorio bajo una convivencia en armonía (Peña, 2009).

Cuando se ingresa a la universidad, allí también, en el centro del círculo de la planta de la edificación, está el Nakchak. A su alrededor los estudiantes o visitantes se sientan para compartir diálogos de experiencias, dificultades, fortalezas o trabajos que adelantan durante el tiempo entre clases. Es un ritual que no permite olvidar que casa y universidad son espacios de conocimiento y de unión para aquellos que se encontraban momentáneamente separados.

De manera que la estructura del sombrero y de la universidad retoman elementos que se hallan múultilocalizados tanto física como míticamente en la cultura Misak. Ya se mencionó la forma del sombrero y el centro del mismo simbolizando el fogón. Ahora recuérdese que el sombrero es tejido en espiral. Pues bien, en el piso de la universidad, ocupando una gran parte del diámetro del círculo central del edifico, se observa en el suelo el caracol en espiral dibujado y una parte de la misma está dibujada bajo el nakchak. El caracol es un eterno retorno (ver figura 2). En ella se marca, en su comienzo, el numisak o llegada (nacimiento), y a medida que los círculos del caracol se despliegan significa para los Misak avanzar en la vida y relacionarse con el futuro, en torno al desarrollo de la familia, de la comunidad y de cada proceso de aprendizaje que debe ocurrir a lo largo de la vida hasta llegar al viaje espiritual (la muerte).

Este proceso de conocimiento se persigue en la Misak Universidad al brindar una educación que se complementa desde diversos saberes y conocimientos, aplicables, expandibles, dispuestos a la retroalimentación desde lo local a lo general o global. Cada ejercicio, práctica o minga propuesta comprende las enseñanzas de los mayores, la naturaleza y las externalidades (entendidas como lo que escapa a la cosmovisión indígena, los conocimientos, costumbres y cultura de los llamados, en algunos casos, "blancos" o "blancomestizos") que forman parte de la cotidianidad Misak, y deben ser interpretadas e 
implementadas de la manera más acorde con su pensamiento y realidad.

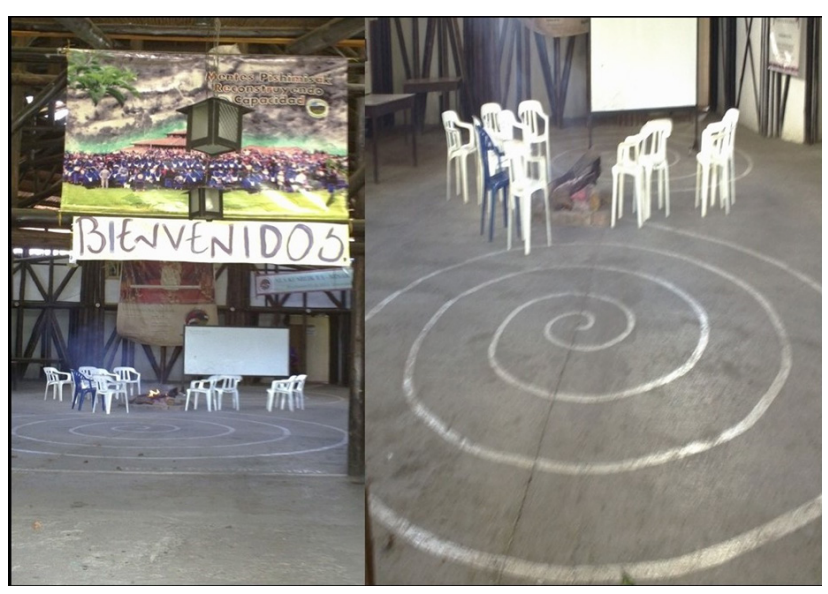

Figura 2. La imagen permite apreciar la espiral del caracol en el suelo que cruza bajo el nakchak

En torno a ese gran círculo, en el que se encuentra el nakchak y el dibujo del caracol, están los salones de clase. (Es importante mencionar que para los Misak el lugar de aprendizaje no son solamente los salones y el espacio físico que enmarca la universidad, sino la naturaleza misma. Incluso en los sueños reciben información, y se convierten en otros escenarios de aprendizaje cuando son compartidos con los mayores para su interpretación). En ellos tienen lugar las asignaturas, materias, cursos o núcleos temáticos, que en la Misak Universidad se nombran como fundamentos, conformados por cuatro ejes: Deber y Derecho Mayor, Administración Propia, Economía Propia, y Organización Sociopolítica. El Deber y Derecho Mayor se refire al deber del cuidado y protección de la naturaleza y sus espíritus, así como a los legados ancestrales y el derecho a la recuperación del territorio, de poseerlo y compartirlo por ser los primeros habitantes del continente americano. A partir de ahí se desglosa la Administración Propia que alude a la facultad de planear, proponer, gestionar, ejecutar proyectos o propuestas de acuerdo a su visión de mundo; la Economia Propia, que aborda también el sistema económico mundial y hace un paralelo con la forma en que los mayores manejaban la economía; y la Organización Sociopolítica para, desde su cosmovisión, garantizar dicho deber y derecho mayor. Cada eje tiene una duración de un año, y el año 5 es dedicado a la profundización que cada estudiante decida realizar en uno de los anteriores ejes.

Cada fundamento está ligado con la cosmovisión o visión de mundo Misak. Son saberes propios pero también se incluyen conocimientos de otros pueblos de América Latina y del mundo, buscando un equilibrio entre los legados ancestrales y los conocimientos generados en la modernidad. Es decir, vinculan lo propio sin desconocer lo global.

La importancia de constituir una estrategia que logre abarcar los conocimientos y saberes ancestrales sin desconocer otras fuentes se concibe, en palabras de un mayor Misak, gracias a la relevancia de la intraculturalidad, alusivo a la identidad auténtica, única y milenaria; a la multiculturalidad concerniente al reconocimiento del otro; a la interculturalidad desde un ámbito recíproco, solidario, consciente y con convicción; y transculturalidad vista como el respeto, cuidado y defensa del planeta (Muelas, sf).

Las clases se desarrollan con la asistencia de los colaboradores provenientes de diversos sectores, que pueden ser titulados o no, en una programación que el coordinador académico diseña para cada fin de semana, tiempo de encuentro por las dinámicas de trabajo de la mayoría de estudiantes, aplicando los conocimientos en cada una de las actividades (en la huerta, minga, salones...).

Para ello cada fundamento se maneja con un sentido de transversalidad que permita el proceso de una manera complementaria. Durante el primer año se trabajan los saberes propios. En el segundo año estos saberes se abordan vinculando a la comunidad, adquiriendo experiencia en la interacción con el cabildo, tatas, programas de educación, siembras, comunicación y otros. El tercer año se enfoca en la labor investigativa y de diagnóstico con la orientación de personas externas (docentes de otras universidades), con el fin de logar implementar técnicas y tecnologías propias ligadas a otras de fuera. Para el cuarto año se direcciona la estructuración y organización del proyecto en el que cada estudiante se esté ocupando, 
comúnmente conocido como trabajo de grado. Ya el quinto año se destina para la profundización; se aborda de fondo el trabajo investigativo que deberá presentarse en lengua materna y español, en caso de pertenecer a una comunidad indígena, sumado a una sustentación de treinta minutos que evidencie el dominio de las dos lenguas, más la interiorización de los fundamentos o ejes (conversación con taita Trino Morales Tombé, julio de 2016).

Los fundamentosquehacenpartedelciclode estudios están fuertemente ligados a los cuatro pilares que cimientan la vida del Misak: 1.articulación al plan de pervivencia y crecimiento Misak, 2.Pensamiento social y cultural, 3.Organización territorial y sociopolítica y 4.Derecho de reconstrucción económica y social. Estos pilares contienen la cosmovisión, territorio y pensamiento Misak que buscan garantizar que la identidad, dignidad, autonomía y autoridad estén presentes durante los ciclos de vida: la preconcepción, concepción, llegada, niñez, juventud, adulto, mayor, viaje espiritual y regreso espiritual pero que también se hagan partícipes de las prácticas culturales, conocimientos y aprendizajes relacionados con la alimentación, sistemas agrícolas, animales, plantas, tierras, astros, aguas, fuegos, minerales, tecnologías, viviendas y diseños, atuendos y diseños, músicacantos, danzas, espíritus, ritualidades y lenguajes, contenidos en una estructura comunitaria que engloba a las personas, núcleo casa (familia: abuelos paternos y maternos, padre, madre e hijos), gran casa o casa grande (territorio, comunidades y pueblo-nación) (Muelas \& Tunubalá, 2009).

Lo anterior está ligado con el equilibrio y armonía entre el ser humano y la naturaleza fundamentado en cuatro principios que a su vez indican el camino para la generación de pensamiento sobre el conocimiento Misak: Morep (escuchar), Aship (ver), Isup (pensar) y Marep (hacer) (Aranda,Tunubalá, Salazar, Rodríguez, Aristizábal, Díaz, 2010). Estos principios a su vez están contenidos en la espiral del caracol, debido a que los mismos se vinculan con las diferentes etapas de crecimiento biológico y espiritual del individuo: cuando se es niño se escucha, cuando se es adolescente se escucha y se ve, y cuando se es joven, adulto y mayor se piensa y se hace (conversación con taita Trino Morales Tombé, julio de 2016), entendiendo lo anterior no como limitaciones, restricciones, sesgos o falta de capacidad para realizarlos en cada etapa sino como una profundización de estos principios que se alimentan de la sabiduría ancestral y de su legado durante la transición de niño a mayor.

Con ese sentido de integralidad como base de la educación el proyecto de educación propia universitaria Misak se enfoca también en realizar una proyección social a través del vínculo con otros centros educativos, comunidades, colegios, convenios de apoyos académicos nacionales e internacionales, participación de población estudiantil colombiana y de otros países, mayores, docentes propios, docentes de comunidades indígenas, docentes de demás universidades del país o mundiales, que estén interesados en vincularse al proceso que continúan alimentando el ejercicio y el trabajo que inició hacia el año 2011.

Algunos de esos convenios de la Misak Universidad implican a universidades como la Universidad Libre de Santiago de Cali, Universidad del Cauca, Universidad del Valle, Universidad Ixil de Guatemala, y se encuentran adelantando gestiones de trabajo con universidades de Bolivia y Perú (conversación personal con taita Tino Morales, coordinador académico de la Misak Universidad). Esta colaboración busca la posibilidad de que estudiantes y docentes de la Misak Universidad viajen a esos lugares, y a su vez permitir la visita y el intercambio de conocimientos de estudiantes y docentes de esas universidades.

En la Misak Universidad, que hasta finales del año 2016 contaba con un número de 78 estudiantes, no existe la valoración por notas, un número o letra. El estudiante cursa los cuatro fundamentos y, en el caso de que en el año cinco al presentar su trabajo de grado se evidencien falencias en alguno de los fundamentos o en el manejo de la lengua, deberá hacer trabajos de acompañamiento a otros estudiantes. De esta forma subsanarán esas debilidades y se ampliarán y fortalecerán sus conocimientos. 
Para ingresar sólo se requiere de la aprobación de la comunidad a la que se pertenezca, tampoco es necesario ser indígena. Ala Kusreik Ya es una universidad indígena abierta, es flexibe con los indígenas que en el curso de sus estudios buscan continuar los mismos en otras universidades; la misma flexibilidad existe con aquellos o aquellas que hayan hecho parte de alguna universidad y deseen ingresar a la Misak Universidad. Pese a no contar con el reconocimiento del Ministerio de Educación, el trabajo continúa gracias al aval de las autoridades indígenas y la disposición de la comunidad que se ampara en su ejercicio autónomo (conversación con el taita Trino Morales Tombé, septiembre de 2016).

\section{Conclusiones}

Los saberes ancestrales y la visión de mundo del pueblo Misak son entrelazados en sus diseños educativos junto con los saberes no indígenas, con el fin de garantizar la pervivencia de su cultura e identidad sin relegarse o aislarse de las problemáticas mundiales. Se trata de continuar andando en el caracol, enrollando y desenrollando en el pensamiento de los mayores la comunidad, la vida, la espiritualidad, la naturaleza y el conocimiento; justamente la propuesta de la Ala Kusreik Ya-Misak Universidad. Con su variedad de dimensiones de análisis desde lo mítico hasta lo arquitectónico, pasando por lo curricular, lo pedagógico, lo artesanal, lo lingüístico, lo filosófico, en fin, es una universidad que vincula bajo sus propias reglas un universo propio.

Estos esfuerzos y constantes demostraciones de trabajo, autonomía, soberanía y resistencia de las comunidades Misak por retornar los saberes usurpados, menospreciados y considerados obsoletos representan un notorio sentido de organización cuando de recuperar la identidad y la cultura se trata. En la misma medida abren una invitación a repensar el sentido y direccionamiento de la educación reglamentada por el Estado colombiano, aunque es necesario aclarar que en las últimas décadas, en escenarios públicos y privados, se vienen gestando estrategias educativas más holísticas y flexibles.
La Misak Universidad, como ya ocurre en otros lugares de Colombia y Latinoamerica, es un ejemplo de sistema educativo que habla de interculturalidad, transculturalidad, multiculturalidad e intraculturalidad en constante renovación-valoración. $\mathrm{Su}$ disponibilidad a nutrirse de otros saberes le permite entrar en un ejercicio de revisión constante en procura de su crecimiento y bienestar integral sin dar cabida a la discriminación ni olvidar su visión de mundo. El mismo debe servir de inspiración y motivación en otros escenarios para que se animen a pensar el tema educativo de forma más transversal, más allá de su reconocimiento o clasificación de algún tipo, amparado en una resignificación del sentido de equilibrio ser humano-naturaleza.

En el Cauca la lucha es notoria. El proceso ha iniciado y continúa en construcción, deconstrucción y reconstrucción. Ese es el sentido de la educación propia: incluir los saberes compartidos de generación en generación, seguir trabajándolos, comprendiendo que son inacabados y manteniéndolos en la vida de los pueblos, sin el desconocimiento u omisión de lo avalado global y científicamente. Se trata de un sentido de complementariedad, no de competitividad.

El reto para la Misak Universidad es mantenerse, defender sus principios y fundamentos afianzando las alianzas con diversos sectores educativos/ sociales que aporten a su crecimiento, y desde los cuales también contribuya con sus saberes; así como la intención de continuar promoviendo estrategias que involucren a niños y jóvenes que se han ido alejando del sentir indígena y su cosmovisión. El nuestro, continuar dando viabilidad a estos ejercicios $\mathrm{y}$ aplicarlos en el quehacer docente en mutua colaboración con todos los actores implicados sin deslegitimar o menospreciar otros campos de saber, sin caer en sesgos academicistas. Recordemos que la educación, dice Juan Muelas Trochez", "ha hecho creer que la ciencia y su método ha incrementado

\footnotetext{
4. Juan Muelas Trochez es un integrante del pueblo Misak, hijo del taita Lorenzo Muelas Hurtado, uno de los fundadores del CRIC y AICO (Autoridades Indígenas de Colombia), también gobernador indígena de Guambía en 1985. Muelas Trochez es uno de los tantos indígenas que busca rescatar y mantener el pensamiento ancestral y la identidad de los pueblos indígenas, especialmente Misak.
} 
conocimientos, cuando eso significa es generación de información masiva que no debe confundirse con sabiduría y conocimientos".

\section{Referencias bibliográficas}

Aranda, L., Perafan, C., Mayorga, E. \& Moreno, S. (2015). Confrontando la cultura hegemónica desde el pensamiento Misak (tesis de maestría). Universidad de Manizales, Popayán, Colombia

Aranda, M., Tunubalá, J. M., Salazar, R. E., Aranda, L. D., Rodríguez, A., Aristizábal, M., Díaz, A. (2010). Parosoto, sembrando cultura ayer, hoy y siempre. Una propuesta educativa propia para la pervivencia del pueblo Misak. Cauca: Universidad del Cauca

Autoridad ancestral del pueblo Misak, Grupo GEIM y UNICEF.(2009). Resignificación del proyecto educativo Misak desde la cosmovisión y las relaciones interculturales para la educación inicial- preescolar (transición) a básica. Un aporte para el sexto planeamiento educativo del pueblo Misak. Silvia

Banco Mundial. (2011). Latinoamérica indigena en el siglo XXI. Washington, D.C.: World Bank Group.

Baronnet, B. (2009). Autonomía y educación indigena: las escuelas zapatistas de las cañadas de la selva Lacandona de Chiapas, México (tesis de doctorado). El colegio de Mexico A.C., Ciudad de Mexico

Bolaños, G. (2012). Motivando la memoria para reconstruir la historia educativa de los pueblos indígenas del Cauca. En: G. Bolaños., V. D. Bonilla., J. Caballero., M. A. Espinoza., V. J. García., J. Hernández, L. Tattay. Nuestra vida ha sido nuestra lucha, resistencia y memoria en el Cauca indigena (Pp. 235-274). Centro de Memoria Histórica, Colombia: Taurus, pensamiento

Bonilla, V. D. (2012). Resistencia y luchas en la memoria Misak. En: G. Bolaños., V. D. Bonilla., J. Caballero., M. A. Espinoza., V. J. García., J. Hernández.,L. Tattay. Nuestra vida ha sido nuestra lucha, resistencia y memoria en el Cauca indigena (Pp. 121-166). Bogotá: Centro de Memoria Histórica, Taurus, pensamiento
Camelo, D. M. (1993). La indumentaria como elemento de identidad y comunicación intraétnica: el caso guambiano. Revista Texto y contexto, 22, 143-157.

Castillo, E. \& Triviño, L. (2008). Historias y trayectorias de maestras y maestros indígenas en el Cauca: ¿la etnoeducación, posibilidad para Otras educaciones? Revista Educación y Pedagogía, 20, 81-97.

Chavez, M (comp) (2011). La multiculturalidad estatalizada. Indigenas, afrodescendientes y configuraciones de estado. Bogotá: Instituto Colombiano de Antropología e Historia.

CRIC. (2004) ¿Qué pasaría si la escuela...? 30 años de construcción de una educación propia. Bogotá: Editorial fuego azul

CONTCEPI. (2013). Perfil del sistema educativo indígena propio S.E.I.P. Bogotá: perfil SEIP

Dagua, A., Aranda M. \& Vasco, L. (2015). Guambianos: hijos del aroiris y del agua. Historia y tradición guambianas. Popayán: Universidad del Cauca.

Demera, J. D. (2005). Misiones religiosas y articulaciones étnicas en el resguardo indígena de Guambia. En A. Bidegain \& J. D. Demera. Globalización y diversidad religiosa en Colombia. (pp. 357-374). Bogotá: Universidad Nacional de Colombia.

De Sousa, B. (2010). Descolonizar el saber, reinventar el poder. Uruguay: Ediciones TRILCE.

Muelas, J \& Tunubalá, F. (2009). Segundo plan de vida de pervivencia y crecimiento Misak Mananasrøkurri Mananasrønkatik Misak Waramik. Bogotá: Dígitos y Diseños

Muelas, J. Elementos para alimentar la re-existencia del Misak-Misak. II foro internacional de Educación Superior Inclusiva. Colombia aprende, la red del conocimiento. Recuperado de http://www. colombiaaprende.edu.co/html/mediateca/1607/article-174759.html 
Muelas, L. (2005). La fuerza de la gente. Juntando recuerdos sobre la terrajería en Guambia, Colombia. Bogotá: Instituto Colombiano de Antropología e Historia ICANH

Pechené, L. \& Tunubalá, J. (2010). "518 años de resistencia, 200 años de los pueblos". El deber, el derecho de re-existencia y la libertad. Revista Maguaré, 24, 415-426.

Pedraza, Z. (1999). En cuerpo y alma: visiones del progreso y de la felicidad. Bogotá: Uniandes. Depto. de Antropología
Peña, J. A. (2009). En las voces del Pishimisak. Apuntes etnográficos sobre el tiempo Misak. Revista Maguaré, 23, 343-369

Rojas, T. (1999). La etnoeducación en Colombia: un trecho andado y un largo camino por recorrer. Revista Colombia Internacional, 46, 45-59.

Torres, I. (1974). Los inconformes. Historia de la rebeldia de las masas. Cali: Universidad del Valle. 